\title{
La gestión del currícullo por competencias en las instituciones de educación superior
}

\section{Management of the competency-based curriculum in higher education institutions}

\author{
Martínez Avendaño, Jairo; Editor académico Prof. Dr. Carlos Alberto \\ Zúniga-González
}

\author{
Jairo Martínez Avendaño \\ jairomar@gmail.com \\ Universidad Nacional Autónoma de Nicaragua, León. \\ Facultad de Ciencias Económicas y Empresarial, \\ Nicaragua \\ Editor académico Prof. Dr. Carlos Alberto Zúniga- \\ González \\ Universidad Nacional Autónoma de Nicaragua, León, \\ Nicaragua
}

Revista Iberoamericana de Bioeconomía y Cambio

Climático

Universidad Nacional Autónoma de Nicaragua, León, Nicaragua

ISSN-e: 2410-7980

Periodicidad: Semestral

vol. 2, núm. 4, 2016

czuniga@ct.unanleon.edu.ni

Recepción: 16 Enero 2016

Aprobación: 14 Diciembre 2016

URL: http://portal.amelica.org/ameli/journal/394/3941751005/

DOI: https://doi.org/10.5377/ribcc.v2i4.5927

Autor de correspondencia: jairomar@gmail.com
Resumen: En Nicaragua, como en el resto de los países del mundo, las universidades, como todas las instituciones $\mathrm{u}$ organizaciones, están experimentando transformaciones muy significativas, que tienen que ver con todas sus funciones sustantivas, pasando por lo académico para llegar a su contexto organizacional. Con estos escenarios, las universidades de hoy deben poder adaptarse a las nuevas demandas y demandas del mercado, el progreso tecnológico y los constantes cambios de los sistemas políticos, además de esto, las universidades nicaragüenses y principalmente las universidades públicas deben enfrentar este proceso de adaptación en una contexto de matriculación masiva sostenida, lo que hace que la tarea sea aún más complicada. La forma de gestionar un currículum en este nuevo contexto, es el tema que se aborda en el presente ensayo, que se desarrolla de tal manera que, al principio, conceptualizamos el término gestión o, mejor dicho, lo contextualizamos en un sentido amplio, para luego dar paso a la gestión del cambio específicamente en el campo de las universidades. Dado que nuestro principal interés es la gestión del plan de estudios, la última parte del desarrollo de la escritura está dedicada a abordar este tema, haciendo hincapié en la gestión del currículum por competencias. No podemos dejar de hacer algunos enfoques concluyentes sobre el tema tratado, para lo cual el ensayo finaliza con la presentación de nuestras conclusiones.

Palabras clave: Administración, Planes de estudio Educación superior.

Abstract: In Nicaragua, as in the rest of the countries of the world, universities, like all institutions or organizations, are experiencing very significant transformations, which have to do with all their substantive functions, going through the academic to reach their organizational context. With these scenarios, the universities of today must be able to adapt to the new demands and demands of the market, the technological progress and the constant changes of the political systems, in addition to this, the Nicaraguan universities and mainly the public universities must face this process of adaptation. in a context of sustained mass enrollment, which makes the task even more complicated. The way to manage a curriculum in this new context is the subject addressed in this essay, which is developed in such a way that, at first, we conceptualize the term management or, rather, we contextualize it in a broad sense, to then give way 
to change management specifically in the field of universities. Since our main interest is the management of the curriculum, the last part of the development of the writing is dedicated to addressing this topic, emphasizing the management of the curriculum by competencies. We cannot fail to make some conclusive approaches to the subject matter, for which the essay ends with the presentation of our conclusions.

Keywords: Management, Curricula Higher education.

\section{Definiendo la Gestión}

Se dice que aquellas organizaciones que han apostado por la implantación de modelos de gestión en su quehacer cotidiano, están en condiciones de abordar su crecimiento futuro de una manera más segura, más rápida y más eficaz, por lo tanto el término de la Gestión está estrechamente asociado con los conceptos de eficacia y eficiencia, pero también le debemos sumar el hecho de que para poder gestionar correctamente se debe considerar el enfoque de un planeamiento estratégico.

La Gestión es entonces, un término que se puede definir desde distintos ámbitos de aplicación del mismo, en el mundo empresarial hay quienes hablan de conjunto de políticas, conceptos y prácticas coherentes entre sí, con el propósito de alcanzar objetivos organizacionales de la empresa de manera eficiente y eficaz (Chiavenato \& Villamizar, 2002), otros como Milgrom y Roberts (1993) lo ven como las estrategias que diseñan las empresas para hacer frente a los problemas cotidianos de la misma y tratar de paliarlos en la medida de lo posible. Estos conceptos concuerdan con lo expresado por García de Fanelli (1998) quien plantea que la calidad de la gestión se ve en la capacidad de anticiparse a los hechos que se vienen, en el buen uso de los recursos disponibles, en la preocupación por los servicios que se prestan, y en el ambiente o «clima» institucional que se vive. Para (Casassus, 1999), hablar de gestión es, básicamente, referirse a dos actividades como son la planeación (o planificación) y la administración de lo diseñado, es así que él define gestión como "la capacidad de articular los recursos de que se dispone de manera tal que se pueda lograr lo que se desea". Como vemos, todos concuerdan en que gestionar adecuadamente una institución u organización pasa por la búsqueda de lograr el buen funcionamiento de la misma, resolver las dificultades de su quehacer y al mismo tiempo poder ser capaces de anticipar los hechos futuros considerando la necesidad de mantener una vocación de planeamiento estratégico.

De igual forma, las definiciones anteriores ponen de relieve el hecho de que la gestión tiene que ver con todos los componentes de una organización o institución, en cuanto a la forma en cómo se organiza, cómo articula los recursos, los objetivos y, por sobre todo, la forma en cómo se interrelacionan las personas en la acción, una acción que tiene normas, estrategias, supuestos o modelos a seguir.

Retomando el tema del planeamiento estratégico del que dijimos debe ser una herramienta para la buena gestión, la cual se utiliza para tratar de controlar el mercado en el tiempo inmediato futuro al que estamos, también es una forma de prepararse para el cambio de los procesos económicos, políticos, sociales, culturales y hasta de los educativos, así lo expresa Aguerrondo (2007) cuando dice que "la historia de la planificación, en general y también su aplicación en el campo educativo, muestra que el planeamiento siempre ha tenido como centro la preocupación por el cambio". Del mismo modo, por ser una herramienta desarrollada para la gestión del Estado, su vocación es la de organizar procesos que hagan posible concretar grandes empresas, e instituciones ya sean productivas o de servicio como lo son las universidades.

\section{NotAS DE AUTOR}


Si lo traemos esto a colación con el ámbito de la Educación Superior podríamos decir que una buena gestión está asociada, al rol que juega la institución, pero también al del sistema universitario como conjunto y al del mismo estado donde se opera, sin obviar los cuatro componentes del estado del arte en el campo de la gerencia como son: La Visión, la Planificación, Gerencia de Operaciones y la Calidad del Proceso. Este planteamiento se justifica en el hecho de que el papel de la institución educativa será diferente según sea su naturaleza, es decir si es pública o privada, si tiene una presencia local, territorial o nacional.

Podemos entonces referirnos a muchos estilos o formas de gestión, o como lo plantea (Sotolongo, 2002) considerar la existencia de diferentes procesos de gestión en las instituciones (principalmente las educativas) y esto es así porque no sólo se gestiona un aspecto de las organizaciones, sino que ésta, está en función de los problemas a enfrentar, de la naturaleza de la institución u organización y de la coyuntura en la que esté inmersa. En este sentido, el aspecto que estamos abordando es la gestión del cambio, es decir écómo se puede gestionar una institución en un ambiente de cambio? Y más específicamente se aborda el tema de la gestión de cambio en las universidades, de lo cual nos ocupamos a continuación.

\section{La gestión del cambio en las universidades}

Para (Salinas, 1998), estamos viviendo tiempos verdaderamente extraordinarios, donde a los cambios económicos, al final de la guerra fría y el desarrollo de la tecnología espacial, se han añadido en los últimos años la explosión de las telecomunicaciones, la digitalización de la información y el desarrollo de la biotecnología, lo cual necesariamente conlleva a tener que implementar nuevas formas de gestionar los procesos productivos, comerciales, y por qué no decirlo, hasta educativos por parte de las instituciones u organizaciones.

Específicamente en el ámbito de las instituciones de educación superior, según Sotolongo (2002) la gestión educativa en los momentos actuales está situada en nuevos escenarios, donde las instituciones comienzan a transitar en un nuevo estilo de gestión caracterizado por una serie de cambios y transformaciones en sus estructuras, que van hacia formas más descentralizadas, donde los enfoques cualitativos están sufriendo modificaciones, para darle paso a la calidad total y donde la concepción normativa comienza a tener una orientación hacia los resultados y los procesos. Es decir que, como ya hemos planteado antes, el mundo moderno está cambiando drásticamente, el proceso de globalización, el rápido desarrollo de la tecnología de la información, la competencia desenfrenada, la necesidad de reducir costos de operación, el énfasis en el cliente o usuario, la calidad total y la necesidad de competitividad constituyen poderosos argumentos para justificar una gestión de calidad. Por lo tanto, se habla hoy en día de la necesidad de gestionar todos los ámbitos de las organizaciones o instituciones; de gestionar el talento humano, gestionar los recursos financieros, gestionar los recursos físicos, gestionar los recursos tecnológicos.

Ante todos estos cambios y la consecuente necesidad de realizar una buena gestión, y por lo tanto planificación de todos los ámbitos de las instituciones u organizaciones debo plantearme la siguiente cuestión, ¿cómo deben gestionar las universidades el cambio? O dicho de otra manera ¿cuáles son los procesos que deben cambiar o evolucionar para no quedar aislados en el pasado? Para dar respuesta a estas interrogantes es importante tener claro, primero, a qué cambios o desafíos se enfrentan las universidades, y en segundo lugar, de qué tipo de recursos se dispone y qué tipo de aprovechamiento se le está dando.

Según (Vessuri, Kreimer, Arellano, \& Menéndez, 2010), las universidades se enfrenta a tres grandes desafíos, en primer lugar enfrentar la internacionalización principalmente de los procesos de investigación, en segundo lugar alcanzar la excelencia tanto de nuestros procesos investigativos como metodológicos y por último formular agendas de trabajo que sean relevantes tanto social como políticamente. De igual forma existen planteamientos que se enfocan en los retos principales que tienen las Instituciones de Educación Superior ante todos estos cambios, esos retos se enmarcan en cumplir con eficacia y eficiencia las tres funciones sustantivas de una Universidad como son la docencia, investigación y transferencia de resultados hacia la sociedad (Extensión). Así mismo, es necesario estar claros que para cumplir con las funciones lo debemos hacer considerando el papel importantísimo que juegan hoy en día las Tecnologías 
de la Información y la Comunicación (TIC), ya que indudablemente debemos reconocer que el progreso tecnológico ha sido el factor impulsor de todas las transformaciones que estamos presenciando.

De hecho autores como (Salinas, 2010), plantean que los avances que en el terreno de las telecomunicaciones se están dando en nuestros días están abriendo nuevas perspectivas a los conceptos de espacio y tiempo que hasta ahora habíamos manejado tanto en la enseñanza presencial como en la enseñanza a distancia, es decir, es indiscutible que hoy por hoy estamos asistiendo a una inflación de cursos on-line, de experiencias de aprendizajes abiertos y flexibles en donde las aulas de clases no necesariamente están ubicadas en los campus universitarios, sino que se puede acceder a ellas desde cualquier espacio virtual, por esta razón no podemos obviar la necesidad de tener presentes estos nuevo enfoques en la enseñanza superior.

Por otro lado, también debemos considerar que en los tiempos en los que estamos viviendo, la buena gestión de los recursos con los que se disponen en las universidades, es una condición necesaria para que éstas puedan ofrecer más y mejor educación, para que puedan ampliar la oferta educativa y al mismo tiempo mejorar los niveles de calidad. Ya que si se continúan implementando los métodos tradicionales de enseñanza, corremos el riesgo de no saber si lo que se está enseñando es relevante o no.

Teniendo claros los cambios a los que se enfrentan, las universidades deben iniciar un proceso de transformación que la impulse a poder adaptarse a este nuevo entorno. Entre ellos, se debe enfocar en el cambio metodológico, como posteriormente se estudiará. Modificando el proceso de enseñanza-aprendizaje y la relación profesor-alumno. Esta situación necesariamente debe dar paso a un planteamiento educativo diferente, más centrado en el aprendizaje y en las competencias, que en la enseñanza y en los conocimientos. Es decir, la figura (o el actor principal) del nuevo proceso debe ser el alumno y se tiene que perseguir un aprendizaje a lo largo de toda la vida. El profesor, en este nuevo enfoque actúa como un mediador y lleva una misión de guía y evaluación continuada. Por tanto, en este nuevo contexto, estar educado significaría adquirir conocimientos de un dominio específico, destrezas cognitivas generalmente útiles, así como estrategias y técnicas efectivas para la realización de tareas concretas.

La forma de autoridad o gobierno, los mecanismos de financiamiento y las modalidades que en cada caso asume la evaluación de la calidad, son otros de los aspectos a incluir al momento de enfrentar el cambio. Es decir que, la concepción del proceso de gestión en una institución educativa, debe ser vista con un enfoque sistémico, donde los cambios que se producen en todos los componentes de la universidad, producen interacciones tanto en su contexto interno como en el externo.

Es así que, la Oficina de Cooperación Universitaria (2010) en su estudio “Tendencias Universidad 2020”, propone que para enfrentar con éxito los cambios, las universidades deben impulsar la implementación de sistemas de certificación eficientes que le permitan la modernización de los procesos en sus tres funciones básicas. De igual forma, se destaca que en la actualidad las estrategias universitarias se desenvuelven en un marco de competencia en el que el nivel tecnológico, la empleabilidad de sus egresados y su carácter innovador son aspectos esenciales para poder triunfar, por consiguiente, estos son otros elementos importantes que se deben incluir en los planes estratégicos de las universidades para enfrentar con éxito los cambios actuales y futuros.

También habrá que considerar el tipo de Universidad que debe funcionar hoy en día, ya la Universidad no es una universidad profesionalizante, como lo plantea (Mollis, 2010), en el sentido de que el principal objetivo de la universidad de hoy, no es formar profesionales y administradores con una visión pragmática y estatista, encapsulados en aprendizajes teóricos y conceptuales. Hoy por hoy, la universidad está más llamada a ser una Universidad científica, cuyo impulso principal es ser una universidad investigadora y generadora del desarrollo social, político, económico y cultural, una universidad que es la protagonista principal del cambio, es decir que se debe convertir en el objeto de cambio, no el sujeto a cambio.

Esto hace que nos hagamos planteamientos un tanto diferentes a los ya expresados con anterioridad, ahora nos preguntamos; ¿cómo se debe hacer el planeamiento de la gestión en la universidad, para que ésta se convierta en un sujeto de cambio? Paradójicamente, la respuesta a esta interrogante podría estar en un 
planteamiento realizado hace más de 50 años, según Aguerrondo (2007), en 1958, el Primer Seminario Interamericano sobre Planeamiento Integral de la Educación que se llevó a cabo en Washington del 16 al 28 de junio de 1958, adopta formalmente la siguiente definición de cómo debe planearse la gestión universitaria: El planeamiento de la educación es un proceso continuo y sistemático en el cual se aplican y coordinan los métodos de la investigación social, los principios y las técnicas de la educación, de la administración, de la economía y de las finanzas, con la participación y el apoyo de la opinión pública, tanto en el campo de las actividades estatales como de la privadas, a fin de garantizar educación adecuada a la población, con metas y en etapas bien determinadas, facilitando a cada individuo la realización de sus potencialidades y su contribución más eficaz al desarrollo social, cultural y económico del país.

Con esto se deja claro que la universidad debe estar consciente que para poder realizar una gestión efectiva y eficiente, se han de considerar todas las dimensiones del ámbito en el que se desenvuelve. De igual forma, tal como lo plantean (Lemaitre \& Zenteno, 2012), también se debe estar dispuesto a dejar la visión tradicional de la educación superior para visualizar una más amplia, que reconozca que no sólo se debe tener el enfoque de que las fuerzas sociales, económicas, políticas y culturales tienen influencia directa sobre la institución, sino que también se ejerce influencia en la dirección contraria. Es decir, que la institución educativa (en este caso la universidad) también influye en el desarrollo social, económico, político y cultural.

Así que, uno de los elementos o dimensiones a considerar son los métodos de la enseñanza y el aprendizaje utilizados, tal como lo plantean (Monereo \& Pozo, 2003), cuando expresan que "dado que la universidad es un espacio privilegiado para la producción, distribución e intercambio social del conocimiento, parece necesario comenzar por repensar la enseñanza y el aprendizaje universitarios, en función de esos cambios que se están produciendo en la naturaleza del conocimiento y su gestión social”. Por consiguiente, es justificable entonces que las universidades hayan iniciado, de un tiempo para acá, a modernizar sus procesos de enseñanza incluyendo instrumentos como las tecnologías del conocimiento (TIC), y transformando sus currículos de tal manera que éstos respondan a las demandas modernas del mercado y por lo tanto, tengan pertinencia social, económica, política y cultural. Más aun, cuando la ampliación y fortalecimiento de la vinculación entre la universidad, la empresa y la sociedad se ha constituido en uno de los temas centrales de las políticas científicotecnológicas tal como lo plantea Correa (1995) citado por (Ganfong, Silveira, \& Martorell, 2002).

Es entonces, indiscutible que los planteamientos realizados en el párrafo anterior nos conduzcan a reconocer que en el mundo moderno, específicamente en las universidades, emerja o aparezca lo que se conoce como la "gestión del currículo".

\section{La gestión de currículo en las universidades}

Según (de Zayas, 1996) el diseño curricular en una institución de educación universitaria, debe ser visto como un proceso de organización y planificación de la formación de los futuros profesionales, y como tal, éste tiene su propia dinámica la cual responde a las leyes internas de la universidad y a las condiciones socio culturales del medio en el que se desempeña. Es así que, para que dicho diseño sea un proceso eficiente y eficaz, debe ser entendido como el medio a través del cual se determinan las cualidades a alcanzar por el egresado.

Por lo tanto, hablar de gestión del currículo en el mundo moderno implica también que estamos hablando de la búsqueda continua de la calidad, ya no se trata sólo de controlar o mantener el estado de las cosas en un orden que permita definir metas fijas, lineales e inamovibles, sino que se deben de conjugar muchos elementos para poder mantener la calidad del servicio que se ofrece a la sociedad, tal es así que (Fernández, 2004) plantea que; al igual que una paleta fauvista, la administración o gestión del currículo debe procurar la armonía del mejoramiento continuo, la disciplina, la reducción de costos, el aprovechamiento y reconocimiento de la gente y el ejercicio de la libertad, de tal manera que se puedan exponer y probar las ideas propias en beneficio del crecimiento personal y de las Instituciones de Educación superior, mejorar, por lo tanto, la calidad de la enseñanza en las universidades, se ha convertido en una de las prioridades de la gestión curricular moderna.

En consecuencia, si estamos de acuerdo con el planteamiento realizado, podríamos entonces afirmar que, el diseño curricular de una universidad, como proceso de organización y planificación de la formación 
de profesionales debe responder, además de, a las leyes internas de la propia universidad, también a las condiciones o contexto cultural, político, social, económico e incluso ambiental, en el que se esté desenvolviendo. Es así que como lo plantea (de Zayas, 1996), el diseño curricular no se debe identificar con el proceso de formación del aprendiz, sino que, éste es el proceso mediante el cual se ha de contribuir a formar las cualidades del sujeto que se desempeñará como profesional, teniendo el sujeto formado, como característica principal un profundo sentido innovador, y yo diría que además de innovador debe ser emprendedor, líder, y con una visión holística de las cosas.

Todos los elementos aportados anteriormente me llevan a realizar la siguiente pregunta ¿En qué consiste la gestión del currículo en las universidades? Aunque, para responder a esta pregunta primero es necesario dejar claro ¿qué es currículo? y ¿cómo se diseña? Una aproximación a la respuesta de estas interrogantes la plantea, de Zayas (1996) expresando que: la carrera es el proceso docente que, en su desarrollo, garantiza la formación del profesional. El diseño de la carrera es el proceso de planificación y organización que concibe cómo debe ser el proceso de formación del profesional. Así mismo, el proceso de formación del profesional se apoya en otro al cual sirve, que es el proceso profesional. El proceso profesional es el que desarrolla el egresado en su actuación y comunicación social, en aras de la solución de los problemas a que se enfrenta en su labor. Es así que, de las relaciones entre estos dos últimos procesos se desprenden las leyes del diseño, y a esto podríamos agregar que también la forma en cómo se debe gestionar ese diseño, de hecho cuando se está planificando la forma en cómo quedará estructurado el currículo, ya lo estamos gestionando, puesto que esa planificación se realizará en función de qué queremos lograr o del profesional que queremos graduar.

Ahora bien, hablando específicamente de la gestión del currículo, ya podríamos aventurarnos a expresar que éste proceso consiste, básicamente, en el aseguramiento de parte de las universidades de que los graduados cumplan con los requerimientos necesarios para el ejercicio profesional en condiciones y niveles adecuados. Esto significa que el currículo desde el momento en que se está diseñando debe estar centrado en el sujeto a formar, el estudiante.

Según (Barrón, Navarrete, \& Ferrer-Balas, 2010), la adaptación al modelo actual de enseñanza y gestión universitaria, supone una reestructuración de la formación, la investigación, la gestión, la implicación y la participación de la comunidad universitaria. Desde el punto de vista de la formación, esta adaptación implica la inclusión de contenidos generales en los currículos de las diferentes titulaciones, por ejemplo; si queremos formar profesionales capaces de actuar críticamente en defensa de la sostenibilidad, debemos incluir contenidos ambientales, socio ambientales, podemos también agregar proyectos de trabajo interdisciplinar, entre diferentes áreas y materias, que garanticen la formación de personas pro-activas y participativas, capaces de tomar decisiones responsables. Pero no debemos obviar que esos contenidos generales deben estar acompañados de contenidos más específicos, propios de la carrera que se está ofertando, y el poder concatenar esos contenidos de tal forma que la formación del profesionalizante sea integral, integrada e integradora es también consecuencia de una buena gestión curricular.

Por otro lado, es importante tener claro que no sólo basta con la inclusión de contenidos diferentes, se deben reorientar, de igual forma, las metodologías de enseñanza hacia estrategias que promuevan el pensamiento crítico y creativo de los alumnos, principalmente en el contexto actual de innovación y competitividad en el que existe un vínculo muy estrecho entre la universidad y la empresa, el cual según (Ganfong et al., 2002) no puede ser visto únicamente como el aprovechamiento ocasional de las capacidades universitarias ya instaladas, por parte de la empresa, sino que requiere además de estrategias activas para la construcción conjunta (universidad - empresa) de ventajas competitivas. Es decir, tal como la plantea (Tejada Fernández, 2000) educarse y educar hoy, exige adaptarse cultural, social, laboral, profesional y personalmente al ritmo del cambio y su velocidad, y en el caso de las universidades esa pauta de cambio se la están dando siempre las empresas, el mercado en su conjunto y la sociedad en general.

Esta vinculación universidad - empresa, de la que hemos hablado en el párrafo anterior, es la que ha permitido la entrada de una nueva metodología para formar profesionales universitarios, los cuales no solo 
cargan un cúmulo de conocimientos de diferentes áreas de las ciencias que estudian, sino que también son capaces, al egresar de la universidad, de transformar ese conocimiento en hechos concretos, es decir, hoy por hoy la estrategia es formar profesionales competentes, propensos al cambio, y dispuestos a dar soluciones concretas a problemas concretos. Esa metodología de formación o si se prefiere, modelo de formación, es lo que se conoce como el "currículo por competencias", de lo cual nos ocupamos de poner en contexto a continuación.

\section{La gestión del currículo por competencias}

Como ya se ha venido planteando, actualmente existe una gran preocupación en las autoridades universitarias y también en todos sus miembros por dar la mejor atención desde el mundo académico a las demandas del sector empresarial y a los requerimientos de los empleadores, lo cual necesariamente genera una búsqueda de nuevas formas de dar respuesta a esas necesidades, esto también conlleva una revisión de la función de la universidad en la sociedad actual y un replanteamiento de los diseños curriculares tradicionales por otros más novedosos, que sean capaces de adaptarse al ritmo de cambio de las nuevas formas y concepciones de procesos productivos, culturales, de relaciones sociales, económicas e industriales.

Consecuentemente como una alternativa ventajosa, para enfrentar estos nuevos retos, ha surgido la implementación del currículo basado en competencias, que como lo plantea el Grupo Operativo de Universidades chilenas (2009) esto es una forma de establecer un aprendizaje más activo, centrado en el estudiante y fundamentalmente orientado a la práctica profesional, ya que, tal como lo expresa (Barrio \& Vásquez, 2006), el cambio social y tecnológico trae consigo un cambio en la organización del trabajo, debido a la casi extinción del ejercicio profesional individual.

Antes de entrar en detalle sobre la forma en cómo se debe gestionar el currículo desde esta nueva perspectiva, es importante tener una clara definición de lo que significa el término "Competencia”, así como el contexto en el que es introducido al ámbito de la educación superior.

Desde el punto de vista conceptual, el término "competencia" tiene muchas acepciones que no necesariamente se refieren al ámbito en el que lo estamos empleando en este escrito, por ejemplo, se puede definir como "rivalidad o lucha entre dos o más personas en condiciones similares para conseguir una misma cosa o superar al rival", por otro lado el término es referido a la "competencia que tienen dos o más empresas para producir y vender un mismo producto en condiciones similares de mercado" esta definición es más propia del ámbito empresarial productivo, también se conceptualiza como "la capacidad de la persona que es competente, que realiza su trabajo o desempeña su función de modo adecuado", este significado es el que ha sido retomado por las universidades para generar los procesos de formación en la actualidad.

Según (Díaz Barriga, 2006) podemos reconocer dos puntos de influencia específicos para el empleo del término competencia en educación. Uno proviene del campo de la lingüística, el otro del mundo del trabajo. Desde el punto de vista del ámbito laboral (que es el ámbito de influencia que nos interesa contextualizar) se concibe el término como una estrategia que se apoya fundamentalmente en el análisis de tareas, a partir de la cual se busca determinar las etapas puntuales en las que se debe formar a un futuro profesional, es decir, esto implica la adquisición de habilidades y destrezas que le vayan a permitir desempeñarse con eficiencia en su labor profesional, he aquí que para este autor el término competencia guarda una relación bien estrecha con los de aptitudes y habilidades.

Por otro lado, en cuanto a la forma en cómo se introduce el término en el ámbito educativo, (Martínez, 2008) afirma que, la significación de capacidades es la vía más empleada para la inclusión de este concepto en la educación y se relaciona directamente con el "saber - hacer" por el estudiante o el egresado de una profesión determinada. Así mismo, también se plantea, que adoptar el modelo no es más que la respuesta generada por los círculos universitarios hacia las demandas del sector productivo y a los requerimientos de los empleadores, los cuales cada vez más requieren de profesionales competitivos capaces de hacer valer sus habilidades en una sociedad cambiante. 
Para (Tobón, 2007), algunos de los argumentos a considerar para tener en cuenta el enfoque por competencias en la educación superior son: a) el enfoque contribuye a aumentar la pertinencia de los programas educativos, en el sentido de que buscan orientar el aprendizaje según los retos y problemas actuales de la sociedad y el mundo empresarial; b) el enfoque posibilita gestionar la calidad de los procesos de aprendizaje de los estudiantes, ya que desde el principio (con el diseño del currículo) se puede definir claramente cómo vamos a evaluar la calidad del desempeño del egresado y cómo vamos a evaluar la calidad de la formación brindada por la institución educativa; c) la formación por competencia se ha convertido en una política educativa internacional de amplio alcance; d) el enfoque de las competencias propicia un ambiente favorable para generar la movilidad de estudiantes, docentes, investigadores y profesionales entre países. Todos estos argumentos son válidos para justificar la incursión de las competencias en los modelos educativos de las universidades.

Es así que, por ejemplo, en Europa a finales del siglo XX inmersos ya en la "Era del Conocimiento", se comienza a tomar conciencia de la importancia que tiene para el desarrollo tanto cultural, social, como económico de todo el continente, incrementar la competitividad de sus ciudadanos a través de una mejora en los sistemas de enseñanza superior. Por tal motivo, en 1998 se firma lo que se conoce como la Declaración de la Sorbona, en donde ministros de educación de cuatro países (Francia, Alemania, Italia y Reino Unido) toman el acuerdo de iniciar un proceso político de cambio a largo plazo de la Enseñanza Superior en Europa. Este acuerdo da inicio al proceso de construcción del Espacio Europeo de Educación Superior (EEES) el cual posiciona a la formación superior como un componente indispensable para consolidar y enriquecer el entorno europeo y al mismo tiempo enfatiza a la necesidad de aumentar la compatibilidad y la comparabilidad de los sistemas de educación superior de todos los países (Broncano \& HEREDERO, 2010). Todos estos procesos conllevaron a que las universidades europeas incluyan cambios metodológicos de la enseñanza aprendizaje, así como también cambios en la relación profesor - alumno, el cual está más centrado en el desarrollo de capacidades, es decir, competencias.

Ahora bien, se debe tener conciencia que frente a esta nueva opción (el currículo basado en competencias), como en toda innovación, también nos vamos a enfrentar con muchas dificultades para implementarla correctamente. De hecho, teniendo conocimiento de causa, puedo decir que las universidades se han encontrado con diversas dificultades en el desarrollo e implementación del modelo curricular basado en competencias. Dentro de los temas más dificultosos que podamos resaltar están la evaluación de los aprendizajes, la integración al currículo de la formación en competencias genéricas o transversales tales como los valores, el liderazgo, las actitudes y otras. Es más, según (Díaz Barriga, 2006), la dificultades pueden aparecer desde el mismo momento en que se está diseñando el currículo, ya que para él, "en el proceso de construcción de los planes de estudio se requiere elaborar una especie de mapa de competencias, el cual sólo se puede hacer a partir de un análisis de tareas", por lo que, si no se tiene el cuidado de realizar el ejercicio correctamente vamos a tener un currículo mal diseñado, que posteriormente también presentará problemas al momento de su ejecución. Por lo tanto, ante estas dificultades debe jugar un papel muy importante la buena gestión que se pueda hacer del proceso desde el inicio del mismo.

Estas dificultades no solo se dan en la integración de las competencias al currículo, sino también en la capacidad de las universidades para proveer la práctica profesional, es decir, en la aplicación real de los conocimientos de su especialidad durante los estudios, muchas veces las competencias sólo están redactadas en los documentos curriculares, pero no se hace un proceso efectivo de desarrollo de las mismas ya que los métodos de enseñanza siguen siendo los tradicionales donde el maestro enseña y el alumno aprende mucha teoría, sin tener la posibilidad de poder ejercitar o llevar a la práctica lo aprendido.

Todo lo planteado en el párrafo anterior se fundamenta en el hecho de que para poder hacer frente a las dificultades encontradas, las universidades deben disponer de una forma eficiente para transmitir el conocimiento, ya que como plantea (Monereo \& Pozo, 2003) las formas en que se produce, difunde e intercambia el conocimiento en una sociedad están íntimamente ligadas a las tecnologías del conocimiento 
dominantes en esa sociedad, que no solo actúan como vehículo de ese conocimiento, sino que lo conforman y determinan la naturaleza de los saberes socialmente válidos y las instituciones sociales que lo gestionan, por lo tanto, si no se cuenta con esas tecnologías del conocimiento, no se podrá cumplir con los objetivos planteados.

En oposición a las dificultades que hemos resaltado trae consigo el modelo, para otros autores la formación basada en competencias implica una serie de ventajas importantes para la educación superior, por ejemplo, permite expresar mejor las capacidades que tienen los egresados al momento de completar sus estudios, esto necesariamente, debe facilitar la inserción al mercado laboral del nuevo profesional. Así mismo, tal como lo hemos expresado anteriormente a partir de los planteamientos realizados por Tobón, el modelo de las competencias propicia una mejor y más efectiva interrelación entre la Universidad y la empresa, la Universidad y el Estado, y la Universidad y la sociedad.

Así mismo, hay quienes plantean que la implementación del modelo propicia la gestión de la calidad, es decir, el enfoque de las competencias posibilita gestionar la calidad de los procesos de aprendizaje de los estudiantes mediante dos contribuciones: evaluación de la calidad del desempeño y evaluación de la calidad de la formación que brinda la institución educativa. Respecto al primer punto, hay que decir que las competencias formalizan los desempeños que se esperan de las personas y esto permite evaluar la calidad del aprendizaje que se busca con la educación, debido a que toda competencia aporta elementos centrales que están en la línea de la gestión de la calidad, tales como criterios acordados y validados en el contexto social y profesional, identificación de saberes y descripción de evidencias. En segundo lugar, el enfoque de las competencias posibilita una serie de elementos para gestionar la calidad de la formación desde el currículo, lo cual se concretiza en el seguimiento de un determinado modelo de gestión de la calidad que asegure que cada uno de sus productos (perfiles, mallas, módulos, proyectos formativos, actividades de aprendizaje, etc.) tenga como mínimo cierto grado de calidad esperada.

Ahora que tenemos una mayor claridad de la forma en cómo llegan las competencias a la educación superior, así como las ventajas y desventajas de poner en práctica el modelo, nos centramos en el tema de la gestión del currículo por competencias, ya que como lo hemos planteado previamente al ser un enfoque novedoso debe implicar también la implementación de nuevas formas de gerencia del proceso de enseñanza - aprendizaje, del diseño del mismo y hasta de los métodos utilizados para convertir el conocimiento en competencia, habilidad y destreza.

Para (Martínez, 2008) la buena gestión debe comenzar desde el primer momento del diseño del currículo, ya que según su planteamiento, un currículo por competencias implica establecer los fines de la formación en términos del "saber hacer de manera efectiva en un contexto dado", así como la necesidad de modelar una actuación en la que estén presente habilidades, conocimientos, destrezas y actitudes que se correspondan con las tareas o funciones a cumplir o ejercer por parte del graduado en la empresa. Más aun, cuando tenemos claro que los desafíos a los que se enfrentaran los nuevos profesionales son de múltiples dimensiones y diferentes contextos, en donde sólo se puede ser capaz de competir teniendo una formación de amplio perfil.

Por otro lado, (Silva, 2006) plantea que en el momento del diseño o transformación curricular, tan importante es diseñar un buen currículo como lo es preparar a los profesores que lo van a ejecutar, ya que, según él, si el currículo llega a los profesores que lo van a aplicar sin que éstos hayan participado en el proceso de diseño, entonces cada uno de los profesores lo adecuará a sus propias vivencias, en correspondencia con su formación pedagógica y profesional, y el resultado seguirá siendo el mismo que antes en líneas generales. Esto ocurre de esta forma, ya que cada profesor universitario actúa según su experiencia precedente la cual con mucha frecuencia, refleja la forma en que a él le fueron impartidos esos contenidos y por lo tanto aunque teóricamente (a través del macroprograma, del microprograma y de las normativas internas de la institución de educación superior) se plantee que la universidad está desarrollando un modelo de formación por competencias, lo que se está haciendo es lo mismo de siempre, transfiriendo conocimientos teóricos, a través de conferencias magistrales, donde el actor principal es el docente y el alumno absorbe información sin desarrollar capacidades. 
Otros de los elementos que se deben tener en cuenta en la gestión del currículo por competencias es que, éste necesariamente implica un cambio metodológico, es decir un cambio en la forma en cómo se va a desarrollar el proceso enseñanza-aprendizaje, para (Broncano \& HEREDERO, 2010) este cambio en la forma de enseñar se debe estructurar de manera tal que el docente no sea la fuente de la que se obtiene el conocimiento, sino que debe ser un facilitador o guía que oriente cómo y de qué manera debe aprender el alumno. En definitiva tanto la labor del profesado como la del alumnado debe cambiar, ya que el primero pasa de ser actor a diseñador de situaciones y escenarios de aprendizajes y el segundo de espectador a actor de su propio aprendizaje.

Necesariamente este nuevo enfoque metodológico conlleva otro aspecto que hay que gestionar de forma diferente en el ámbito educativo, nos referimos al tema de "la evaluación" según (MARGALEF, 2005) el nuevo modelo educativo supone un aprendizaje centrado en el alumno, un fortalecimiento de la autonomía de los estudiantes, así como un desarrollo de habilidades y capacidades críticas, que obligatoriamente requieren de nuevas formas de evaluar que estén acorde a los nuevos modos de concebir la enseñanza. En este contexto la evaluación se debe caracterizar por ser holística, democrática, que cuente con la participación de todos los que intervienen en el proceso mismo, también debe ser una actividad ética que se guíe por la búsqueda de la equidad, debe tener en cuenta, siempre, al alumno y al contexto en que se desempeña, en fin, es una actividad crítica de aprendizaje.

En esta misma línea de la evaluación de los aprendizajes con la implementación del modelo de formación por competencias, también se afirma que éste exige de la comprobación sistemática y final de la efectividad en la ejecución de las tareas por parte del estudiante, tareas que deben acercarse lo más posible a aquellas que deberá cumplir en el ejercicio de su profesión, lo cual nos sitúa, necesariamente, en la evaluación de resultados observables y objetivamente medibles (Martínez, 2008).

Como vemos, hablar de gestión del currículo por competencias, implica tener presente que se deben realizar muchos cambios a lo interno de la institución que ejecuta dicho modelo, es decir, no se trata de un cambio que se produce de forma instantánea, en este caso no basta con legislar o decretar una reforma que nos lleve a una transformación de concepciones y modelos, también es necesario realizar cambios profundos en todos los ámbitos de la Universidad, es necesario incluir en el mismo proceso de rediseño a los actores principales como son los alumnos, docentes, trabajadores, sociedad en general, empresas privadas, y el Estado, de tal forma que al momento de iniciar a ejecutar el nuevo modelo éste sea efectivo, pertinente e innovador.

\section{ConcLuSiONES}

Una vez hemos abordado el tema de la gestión en las universidades, y se ha relacionado éste con el desarrollo de un currículo por competencias, procedemos ahora a plantear algunos elementos conclusivos sobre los mismos, teniendo siempre el cuidado de no pretender dar por finalizada la discusión alrededor de los temas puestos en contexto y más bien contribuir a que la discusión sea enriquecida con mayores elementos discursivos.

En primer lugar debemos tener claro que el término "gestión" es utilizado en muchos ámbitos de aplicación. Pero haciendo alusión específicamente al ámbito de la Educación Superior, podemos decir que una buena gestión estará estrechamente asociada al tipo de institución del que estemos hablando si es pública o privada, al rol que ésta juega en la sociedad, a la forma en cómo obtiene sus recursos (pueden ser fondos públicos, privados, subvencionados) Pero, independientemente de cuál sea la naturaleza de la institución debemos ser conscientes que en el contexto actual la gestión de las universidades está en un proceso de clara transformación, en el cual las decisiones son tomadas de acuerdo a las señales que emitan el mercado, el Estado y la sociedad como tal, por lo tanto la gestión de la educación universitaria se debe estudiar, también desde un contexto de cambio. 
Históricamente la Universidad ha tenido como función fundamental transmitir el conocimiento por medio de la investigación y la formación, pero, en el contexto actual de cambio y transformación, éstas necesariamente están obligadas a formar profesionales competitivos, capaces de desempeñarse con eficiencia y calidad en el mercado laboral, profesionales que no solamente egresen de sus carreras cargados de conocimientos teóricos, sino que más bien sean competentes, afronten el cambio con objetividad y sean sujetos (no objetos) del mismo. Esto solo se logra a través de una buena gestión del cambio, que incluya todos los ámbitos de la institución desde la gerencia de los recursos financieros, pasando por los físicos, tecnológicos, humanos, hasta llegar a la gestión del currículo.

En este ensayo, al abordar el tema de la gestión del cambio nos centramos específicamente en la gestión del cambio curricular, lo cual necesariamente implica una búsqueda constante de la calidad en los procesos de enseñanza - aprendizaje ejecutado por las universidades. Evidentemente, debido a las nuevas demandas del mercado laboral, el cual exige cualificaciones y características muy particulares en los egresados, el proceso de diseño del currículo también debe estar sujeto a cambio. Estos cambios no solamente se deben evidenciar en las materias o componentes curriculares a impartir, sino que también deben realizarse en los contenidos de los mismos, en la forma en cómo se imparten, en la forma en cómo y a quienes incluimos a la hora de diseñarlo, lo cual necesariamente debe encaminarnos a la implementación de nuevas maneras de formar profesionales.

El diseño curricular por competencias es una de las alternativas innovadoras que se ha adoptado para hacer frente a las nuevas y constantes exigencias del mercado. Teniendo en cuenta que innovar implica alterar la realidad vigente, las prácticas comunes y buscar alternativas y modos nuevos de hacer, crear y recrear ideas, podríamos concluir que el propósito específico de la implementación del modelo por competencias es aumentar la posibilidad de transformar las experiencias de aprendizaje en resultados organizacionales basados en desempeño, es decir, asegurar que los aprendices sean capaces de demostrar sus capacidades aprendidas después que hayan adquirido una combinación de conocimientos, habilidades y destrezas.

Para cumplir con este objetivo del diseño curricular por competencias, es necesario crear las condiciones adecuadas para gestionar de forma eficiente este nuevo modelo. En primer lugar, se hace necesario adaptar el sistema universitario hacia los logros de las directrices que permitan a los alumnos la consecución de competencias, esto se hace en el propio proceso de diseño del currículo definiendo situaciones de aprendizaje en las que se favorezca no sólo la adquisición de conocimientos, sino también la de habilidades y actitudes que tributen a generar un perfil formativo amplio.

Las estrategias de enseñanza - aprendizaje son otros de los elementos que debemos adaptar al nuevo modelo, ya la manera tradicional de transferir conocimiento a través de conferencias magistrales, por ejemplo, no es viable en este nuevo contexto, ahora los docentes deben convertirse en guías facilitadores del proceso de aprendizaje y los alumnos son los actores principales de su formación, con lo cual los métodos más viables son aquellos que propician que el estudiante sea el actor principal, por ejemplo, el Aprendizaje Basado en Problemas (ABP), el aprendizaje experiencial, los estudios de casos, entre otros. Es decir, que la estrategia educativa debe basarse en aprender a aprender lo cual debe aumentar la competencia del educando y su capacidad para responder a las demandas del mercado.

Hay quienes plantean que la forma en cómo se produce, difunde en intercambia el conocimiento en la sociedad actual, está íntimamente ligada a las tecnologías del conocimiento que imperan en esa sociedad, por lo tanto, es de vital importancia incluir en los nuevos modelos de formación, como una práctica de buena gestión, esas tecnologías del conocimiento que sirvan de soporte y al mismo tiempo validen y garanticen la pertinencia y durabilidad de los conocimientos, habilidades, destrezas y actitudes adquiridas por el alumno. Estas tecnologías a las que nos referimos pueden ser las redes sociales, las plataformas virtuales, el mismo correo electrónico, los teléfonos celulares inteligentes, en fin, muchas otras más.

Podríamos seguir mencionando muchos elementos más que se deben tener en cuenta para garantizar una gestión del currículo por competencias efectiva y eficiente, tales como la forma en cómo se deben estructurar los planes de clases, qué componentes deben ser incluidos y cuáles no, la manera en cómo los profesores 
deben prepararse para impartir un componente, cuáles son las estrategias de las que deben apropiarse, cómo se deben diseñar los salones de clases para que el alumno no continúe recibiendo el conocimiento de una sola fuente o dirección, en fin muchos aspectos más, pero no lo vamos a hacer, lo último que vamos a concluir es que si queremos que las competencias incidan efectivamente en los estudiantes, resulta indispensable que se promuevan cambios radicales en los otros dos factores que comprenden la triada de todo proceso educativo: el currículo que se imparte y los profesores que lo imparten, es decir, no se puede cambiar la forma de pensar de los educandos, si el educador y el instrumento que utiliza para educar no cambian de visión.

\section{LITERATURA CITADA}

Aguerrondo, I. (2007). Racionalidades subyacentes en los modelos de planificación (educativa). Revista Brasileira de Política e Administração da educação, 23(3), 463-481.

Barrio, G. H., \& Vásquez, O. C. (2006). Aplicación del enfoque de competencias en la construcción curricular de la Universidad de Talca, Chile. Revista Iberoamericana de Educación, 40(1), 3.

Barrón, A., Navarrete, A., \& Ferrer-Balas, D. (2010). [16] Sostenibilización curricular en las universidades españolas. ¿ ha llegado la hora de actuar? Revista Eureka sobre Enseñanza y Divulgación de las Ciencias, 7.

Broncano, S. G., \& HEREDERO, C. D. P. (2010). Análisis y evaluación de la gestión por competencias en el ámbito empresarial y su aplicación a la universidad. Revista Complutense de Educación, 21(2), 323-343.

Casassus, J. (1999). Marcos conceptuales para el análisis de los cambios en la gestión de los sistemas educativos. La gestión: en búsqueda de sujeto. Seminario internacional-Reformas de la gestión de los sistemas educativos en la década de los noventa-Santiago de Chile, 13-14.

Chiavenato, I., \& Villamizar, G. (2002). Gestión del talento humano. de Desarrollo, C. I., \& de Universidades Chilenas, G. O. (2009). Diseño curricular basado en competencias y aseguramiento de la calidad en la educación superior: CINDA.

de Zayas, C. M. Á. (1996). El diseño curricular en la Educación Superior cubana. Pedagogía Universitaria, 1(1).

Díaz Barriga, Á. (2006). El enfoque de competencias en la educación:¿ Una alternativa o un disfraz de cambio? Perfiles educativos, 28(111), 7-36.

Fernández, A. (2004). Universidad y Currículo en Venezuela: Hacia el tercer milenio: Fondo Editorial Humanidades.

Ganfong, C. P., Silveira, J. L. F., \& Martorell, M. P. (2002). Tendencias en la vinculación universidad-empresa en la última década del siglo XX. Pedagogía Universitaria, 7(2).

García de Fanelli, A. M. (1998). Gestión de las universidades públicas: la experiencia internacional.1ed. serie nuevas tendencias

Lemaitre, M. J., \& Zenteno, M. E. (2012). Aseguramiento de la calidad en Iberoamérica. Educación Superior-Informe 2012.

MARGALEF, L. (2005). Los retos de la evaluación auténtica en la enseñanza universitaria: coherencia epistemológica y metodológica. Perspectiva Educacional, Formación de Profesores(45), 25-44.

Martínez, G. L. (2008). El currículo por competencias. Un tema a debate. Pedagogía Universitaria, 13(3).

Milgrom, P. R., Roberts, J., \& Jimeno, E. (1993). Economía, organización y gestión de la empresa: Ariel Barcelona.

Mollis, M. (2010). Las transformaciones de la Educación Superior en América Latina: Identidades en construcción. Educación Superior y Sociedad, 15(1), 11-24.

Monereo, C., \& Pozo, J. I. (2003). La cultura educativa en la universidad: nuevos retos para profesores y alumnos. $L a$ universidad ante la nueva cultura educativa. Enseñar y aprender para la autonomía, 15-30.

Salinas, J. (1998). El rol del profesorado universitario ante los cambios de la era digital. Agenda Académica, 5(1), 131-141.

Salinas, J. (2010). ¿ Qué aportan las tecnologías de la información y la comunicación a las universidades convencionales? Algunas consideraciones y reflexiones. Revista Educación y Pedagogía, 14(33). 
Revista Iberoamericana de Bioeconomía y Cambio Climático, 2016, vol. 2, núm. 4, Agosto-Diciembre,...

Silva, P. H. (2006). El reto de la transformación curricular. Revista Iberoamericana de Educación, 40(3), 5.

Sotolongo, B. R. (2002). Propuesta de un modelo para gestión de la docencia. Pedagogía Universitaria, 7(2).

Tejada Fernández, J. (2000). La educación en el marco de una sociedad global: algunos principios y nuevas exigencias.

Tobón, S. (2007). El enfoque complejo de las competencias y el diseño curricular por ciclos propedéuticos: Universidad de Los Andes (ULA).

Universitaria, O. d. C. (2010). 2020 tendencias universidad: estudio de prospectiva: Oficina de Cooperación Universitaria.

Vessuri, H., Kreimer, P., Arellano, A., \& Menéndez, L. S. (2010). Conocer para transformar: producción y reflexión sobre Ciencia, Tecnología e Innovación en Iberoamérica: UNESCO-IESALC. 